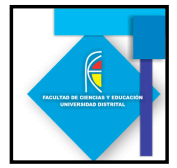

\title{
PERFIL CONCEPTUAL DE EQUILIBRIO A PARTIR DE LAS IDEAS EN COMUNIDADES CULTURALMENTE DIFERENCIADAS: IMPLICACIONES PARA UNA EDUCACIÓN EN CIENCIAS EN UNA SOCIEDAD CULTURALMENTE DIVERSA
}

\section{CONCEPTUAL PROFILE OF EQUILIBRIUM FROM THE IDEAS IN COMMUNITIES CULTURALLY DIFFERENTIATED: IMPLICATIONS FOR A SCIENCE EDUCATION IN A CULTURALLY DIVERSE SOCIETY}

Rosa Inés Pedreros Martínez ${ }^{1}$

\begin{abstract}
Resumen
Desde la década de los años 80 las investigaciones en enseñanza de las ciencias se han centrado en el estudio de las ideas, concepciones y representaciones de los estudiantes. Estudios en los cuales aún persisten interrogantes: ¿Por qué los estudiantes no aprenden lo que se les enseña? y, ¿Por qué no se logra un aprendizaje significativo?

Particularmente, en Colombia cobra sentido tener en cuenta las ideas que circulan dado que está conformado por comunidades culturalmente diferenciadas, que implica una diversidad epistémica y cultural. Así, se formula el problema de investigación ¿Qué interrelaciones se encuentran en las relevancias y compromisos epistemológicos y ontológicos subyacentes a las explicaciones sobre el equilibrio en las ciencias y en dos comunidades culturalmente diferenciadas? La investigación se enmarca en la perspectiva teórica cultural, intercultural, y multicultural de la configuración de los conocimientos, el objetivo es determinar las interrelaciones entre las relevancias y compromisos (epistemológico y ontológico).
\end{abstract}

Palabras Claves: Perfil conceptual, ciencia, cultura, representaciones, diversidad.

\begin{abstract}
Since the early 80 's research in science education have focused on the study of ideas, conceptions and representations of students. Studies in which questions still persist: ¿Why do not students learn about what they are taught? And ¿why not is achieved meaningful learning?
\end{abstract}

\footnotetext{
${ }^{1}$ Doctorado Interinstitucional en Educación Sede Universidad Distrital "Francisco José de Caldas" Departamento de Física Universidad Pedagógica Nacional. rpedreros@pedagogica.edu.co
} 
$5^{\circ}$ CONGRESO NACIONAL DE ENSEÑANZA DE LA FÍSICA.

Universidad Pedagógica Nacional (U. P. N)

Universidad Distrital Francisco José de Caldas (U. D. F. J. C)

Bogotá, Colombia. 16 al 20 de mayo 2011

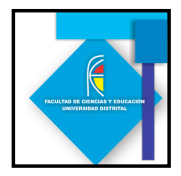

Particularly in Colombia makes sense to take into mind the ideas that circulating because is made up culturally different communities, which implies an epistemological and cultural diversity. Thus, is formulated the research question; What are the relationship in the relevance and underlying epistemological and ontological commitments to the explanations about the balance in the science and in two culturally different communities? The research is part of the cultural theoretical perspective, intercultural, and multicultural knowledge settings, the aim is to determine the interrelationships between the relevance and commitment (epistemological and ontological).

Keywords: Conceptual profile, science, culture, representations, diversity.

\section{Presentación}

En el presente escrito se exponen las reflexiones iniciales que orientan la investigación a propósito de las ideas, concepciones y representaciones; estudios históricos-culturales y contextuales y perfil conceptual.

Como punto de partida tengamos en cuenta que desde la década de los años 80 las investigaciones en enseñanza de las ciencias se han centrado en el estudio de las ideas, concepciones y representaciones de los estudiantes. Los estudios se han centrado en qué hacer con ellas en la clase. Algunas investigaciones son cambios estructurales, (Piaget \& Indelbert, 1972); (Piaget, 1984); (Nussbaum, 1989); cambios en conceptos o nociones individuales -contenidos-, (concepciones alternativas); cambio conceptual en áreas 0 dominios específicos de conocimiento, (Driver \& Erikson, 1983); (Driver, 1986); (Driver, Squires \& Wood-Robinson, 1994); evolución de las concepciones, (Giordan \& De Vecchi, 1988) y, cambio en el contenido conceptual (Teoría de campos conceptuales), (Vergnaud, 1990, 1996, 2007). Al respecto, los resultados de las prácticas para lograr el cambio conceptual, demuestran que a pesar de los esfuerzos realizados aún no se ha alcanzado este objetivo (Duit, 1994, 1999); (Duit \& Treagus, 1998). Persisten interrogantes ¿Por qué los estudiantes no aprenden lo que se les enseña? y, ¿Por qué no se logra un aprendizaje significativo?

Otras investigaciones como cambio de los conceptos componentes y cambio en la forma de conceptualizar (Teorías implícitas); (Rodrigo, 1985, 1994); (Rodrigo, Rodríguez \& Marrero, 1994); (Pozo, Rodríguez, \& Marrero, 1993); (Utges, Jardón, Ferabóli \& Fernández, 2000); (Utges \& Pacca, 1998, 2003); y, comprensión de las representaciones y modelos mentales, (Norman, 1983); (Johnson-Laird, 1983, 1996); (Pozo, 1989, 2003); (Pozo \& Gómez, 1998); (Morrison \& Morgan, 1999); (Moreira \& Greca, 2002, 2003); (Moreira, 1999, 2005) y, (Justi, 2006); (Justi \& Gilberth, 2006), empiezan a contemplar la diversidad, pero no tienen en cuenta el contexto y las comunidades culturalmente diferenciadas. Asunto importante para Colombia, por su diversidad epistémica, conceptual y cultural.

Investigaciones como Colectivos de pensamiento, (Fleck, 1986a, 1986b); "Visiones de mundo", (Cobern, 1993, 1994, 1996; Cober \& Loving, 2001); Perfil Conceptual (PC), (Mortimer, 1998, 2000, 2001) y, Conglomerados de relevancias, (Molina, 2002; Molina, 
$5^{\circ}$ CONGRESO NACIONAL DE ENSEÑANZA DE LA FÍSICA.

Universidad Pedagógica Nacional (U. P. N)

Universidad Distrital Francisco José de Caldas (U. D. F. J. C)

Bogotá, Colombia. 16 al 20 de mayo 2011

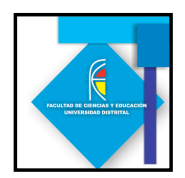

López \& Mojica, 2005), posibilitan el reconocimiento de las comunidades culturalmente diferenciadas y, preguntar por ¿Cuál es el origen y condicionamiento histórico-social en la formación de los conceptos, la organización de la mente y las modalidades de pensamiento y formas de hablar en la clase? y ¿Cómo se presenta la producción de significados?

Lo expuesto se constituye en un asunto relevante para la comunidad de docentes por el reconocimiento de la diversidad cultural, conceptual y epistémica de las comunidades y por la necesidad de una educación intercultural, pertinente para Colombia, por ello se formula la investigación, recogida en la pregunta: ¿Qué interrelaciones se encuentran en las relevancias y compromisos epistemológicos y ontológicos subyacentes a las explicaciones sobre el equilibrio en las ciencias $y$ en dos comunidades culturalmente diferenciadas?, la cual se encuentra en curso.

\section{Reflexiones Iniciales}

A propósito de las ideas, concepciones y representaciones se distingue que sobre las concepciones previas de las personas con respecto a la ciencia y su evolución, se distinguen dos tipos de investigaciones, (1) Promover cambios generales en el razonamiento sin concentrarse en proporcionar conceptos específicos (cambio estructural). (Inhelder \& Piaget, 1972; Piaget, 1984; Nussbaum, 1989). En esta propuesta se supone que las ideas o conceptos construidos por los adolescentes dependen de estructuras lógicas más generales que producen un grado notable de homogeneidad en las ideas mantenidas por los alumnos en diversos dominios o situaciones y, (2) Construir el conocimiento teniendo en cuenta las concepciones de los que aprenden, dicha evoluciona se da a partir de la curiosidad, a través de actividades de comparación con las concepciones de otros y con los hechos. Se considera que la apropiación del saber no es efectiva más que por medio de la evolución paralela de distintos conceptos y por integración progresiva de éstos en el proceso del pensamiento (estadios de integración) llegando a niveles sucesivos de formulación. Estas actividades permiten la formulación de problemas científicos, busca elementos de respuestas, analizarlos, establecer nuevas relaciones entre las adquisiciones puntuales, las cuales están en conexión con las representaciones previas. (Giordan \& De Vecchi, 1987).

La denominada, concepciones alternativas, busca la comprensión de nociones específicas reconociendo que las personas poseen sus propios conceptos -previos a la instrucciónsobre los fenómenos científicos; se piensa en adquisición de las unidades específicas del conocimiento. Promueve cambios en conceptos o nociones individuales, sin preocuparse demasiado por sus relaciones con otras representaciones. (Driver \& Erikson, 1983; Driver, 1986; Driver, Squires, Rushworth, \& Woodo-Robinson, 1994).

En la llamada Teoría de campos conceptuales, se asume el desarrollo cognitivo dependiente de las situaciones y conceptualizaciones específicas para lidiar con ellas. Esta es una teoría psicológica del proceso de conceptualización de lo real que permite localizar y estudiar continuidades y rupturas entre conocimientos desde el punto de vista de su contenido conceptual. (Vergnaud, 1990, 1996). 
$5^{\circ}$ CONGRESO NACIONAL DE ENSEÑANZA DE LA FÍSICA.

Universidad Pedagógica Nacional (U. P. N)

Universidad Distrital Francisco José de Caldas (U. D. F. J. C)

Bogotá, Colombia. 16 al 20 de mayo 2011

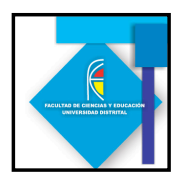

En las representaciones internas (modelos mentales), se busca entender las representaciones de los conceptos científicos y los procesos de construcción que realizan las personas. (Norman, 1983; Jhonson- Laird, 1983, 1996; Moreira, 1994, 1999; Moreira \& Greca, 2002, 2003; Justi, 2006; Justi \& Gilbert, 2006). Otros consideran la redescripción representacional, (Pozo, 1989, 2003); (Pozo \& Gómez, 1998). Y, las teorías implícitas, en la que se considera que las ideas de los alumnos están organizadas dentro de teorías implícitas o personales, con características bien diferenciadas de las teorías científicas. (Rodrigo, 1985, 1994); (Rodrigo, Rodríguez \& Marrero, 1993); (Utges, Jardón, Ferabóli \& Fernández, 2000); (Utges \& Pacca, 2003).

En las primeras investigaciones, se busca la comprensión de las representaciones internas para entender los procesos de construcción, evolución, cambio de las representaciones y lograr el aprendizaje significativo en la clase. En la segunda, se asume que el cambio conceptual sería no sólo un cambio de los conceptos componentes sino ante todo un cambio en la forma de conceptualizar. En estas investigaciones se empiezan a contemplar la importancia de la diversidad en el aula a partir de las representaciones y teorías personales. Sin embargo no muestran la relación entre contexto local y global y no evidencian la presencia de comunidades diferentes en el aula, lo cual implica una diversidad epistémica, conceptual y cultural.

Sobre estudios histórico culturales y contextuales surgen propuestas e investigaciones como la de los colectivos de pensamiento, (Fleck, 1986, 1986b); las visones de mundo, (Cobern, 1993, 1994, 1996); la relación entre modalidades de pensamiento, formas de hablar, (Mortimer, 1998, 2000, 2001) y, conglomerados de relevancia, (Molina, 2000, 2005).

\section{Reflexiones Finales}

La génesis y el desarrollo de un hecho científico, son explicados por las ideas iniciales relativas al hecho, surgidas en el pasado, y que a pesar de las modificaciones, continúan existiendo. Estas ideas van siendo poco a poco modificadas, sufriendo re-interpretaciones de acuerdo con el pensamiento en la evidencia; el pensamiento va modificándose y adaptándose a el medio como sistema. El observar es dirigido, por medio de un condicionamiento históricocultural, siempre teniendo en consideración un concepto preformado; este concepto no es de origen individual, se origina en un colectivo. (Fleck, 1986a, 1986b). Esta propuesta arroja elementos para comprender y reconocer las comunidades culturalmente diferenciadas en países como Colombia, en particular como emerge la relación entre el individuo, el colectivo y la realidad que se vive y, el origen y condicionamiento histórico-social en la formación de los conceptos.

La visión de mundo en la que se origina el constructivismo contextual propuesta por Cobern, (1993, 1994, 1996), corresponde a la organización fundamental de su mente, incluido un conjunto de presupuestos subyacentes a sus actos, sus pensamientos, sus disposiciones, sus juicios, etc. Presupuestos que tienen un carácter tanto ontológico como epistemológico, constituyendo criterios para las apreciaciones de cuales ideas o creencias son válidas y relevantes, es decir, tienen fuerza o alcance para el individuo. Un concepto o 
$5^{\circ}$ CONGRESO NACIONAL DE ENSEÑANZA DE LA FÍSICA.

Universidad Pedagógica Nacional (U. P. N)

Universidad Distrital Francisco José de Caldas (U. D. F. J. C)

Bogotá, Colombia. 16 al 20 de mayo 2011

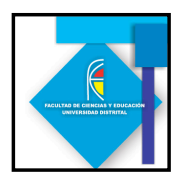

una creencia tiene fuerza, si ella ocupa una posición central, es no marginal en el pensamiento del individuo, caso que se muestra relevante para una gran variedad de contextos.

Se considera la relación entre modalidades de pensamiento y formas de hablar, referidas a la noción de Perfil Conceptual y la investigación de la producción de nuevos significados en las clases de ciencias en la propuesta de Mortimer, (1998, 2000, 2001). Se tiene en cuenta en esta propuesta el presupuesto básico que, en cualquier cultura o persona no existe una única forma homogénea de pensar sino diferentes tipos de pensamiento verbal. Se caracteriza esta heterogeneidad del pensamiento verbal en términos de un Perfil Conceptual que reconoce la coexistencia en el individuo de dos o más significados para una misma palabra o concepto, que se emplean correctamente en diferentes contextos.

En los Perfiles Conceptuales, la evolución conceptual no es entendida como la sustitución de las concepciones previas de los aprendices por ideas científicas, sino como un enriquecimiento del espectro de ideas de que se dispone para la comprensión de un asunto dado. El Perfil Conceptual, es un sistema superindividual de formas de pensamiento que puede tener un individuo en una determinada cultura, tiene un aspecto dual, en donde cada una de las diferentes zonas que lo conforman está definida por su naturaleza ontológica y epistemológica, permitiendo con esto introducir la idea de que cada concepto científico, independiente de la zona en la que se encuentre, presenta una definición ontológica y una definición epistemológica particular. De esta manera pueden existir zonas del Perfil Conceptual cuyos presupuestos epistemológicos sean compartidos pero en el plano ontológico sean disímiles. Se reconoce que el Perfil Conceptual hace parte de un análisis cultural pero no se tienen investigaciones que establezcan una relación con la diversidad cultural de las comunidades existentes y las implicaciones de un análisis intercultural.

Los Conglomerados de Relevancia propuesta de Molina, (2000, 2002, 2004, 2005a), se constituye a partir de la idea de valor como decisión, lo cual permite resolver las ambigüedades que se generan en: (a) los juegos del lenguaje, (b) los actos de habla, (c) los usos metafóricos y analógicos, (d) la negociación del significado y (e) la apertura, no linealidad, y amplias opciones de significar, características que podemos identificar en la concepción de conocimiento inspirada en la metáfora de la red. Estos estudios posibilitan adelantar una interpretación cultural y un análisis intercultural, en particular para la presente investigación sobre los perfiles conceptuales.

Finalmente, las perspectivas expuesta abren nuevas rutas de estudio y desafíos para pensar la ciencia y su enseñanza, en particular la enseñanza y aprendizaje de un concepto como el de equilibrio en el cual cobra sentido la indagación de los principios ontológicos y epistemológicos para comprender los modos de pensar y hablar en el aula, el reconocimiento de la diversidad cultural, el contexto y las comunidades presentes en los espacios escolares al adelantar la acción pedagógica. 

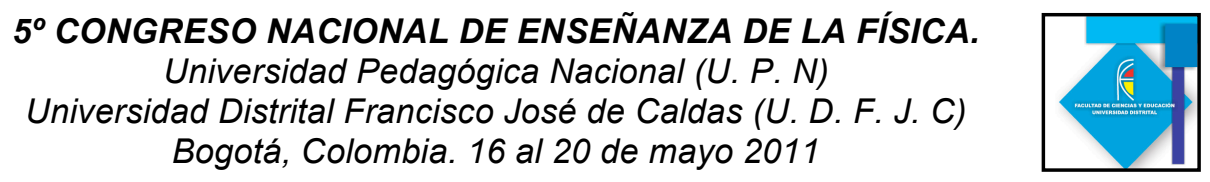

\section{Referencas Bibliográficas}

Alcaldía Mayor de Bogotá y Secretaria de Educación. (2007). Cuadernos de currículo. Bogotá, Colombia: Impresa Nacional.

Cober, W., \& Loving, C. (2001). Defining "Science" in a Multicultural World: Implications for Science Education. Science Education. 85:50-67.

Cobern, W. (1993). College student's conceptualizaions of nature: an interpretative world analysis. Journal of Research in Science Teaching. 30, 8: 985-951.

Cobern, W. (1994). Point: Belief, undertanding, and the teaching of evolution. Journal of Research in Science Teaching. 31: 583-590.

Cobern, W. (1996). Worldview theory and conceptual change . Science Education. 80, 5: 579-610.

Driver, R. (1986). Psicología cognoscitiva y esquemas conceptuales de los alumnos. Enseñanza de las ciencias. 4,1: 3-15.

Driver, R., \& Erikson, G. (1983). Theories in action: some theorical and empirical issues in the study of students conceptual frameworks in science. Studies in science education. 10.

Driver, R., Squires, A., Rushworth, P., \& Woodo-Robinson, V. (1994). Making sense of secondary science. Londres y Nueva York: Routledge.

Duit, R. (1994). Conceptual change. Approaches in science education. Symposium of Conceptual Change. Alemania: Universidad de Jena.

Duit, R. (1999). Concetual change. Aproaches in science education. In: W. Shonotz S. Vosniadou and M. Carretero (eds.). New perpectives on conceptual change. Oxford: Elisevier.

Duit, R., \& Treagus, D. (1998). Learning science: from behaviourism towards social constructivism and beyond. In: B.J. Frase and K.G. Tobin (Eds). Dordrecht: Kluwer Academic Publishers.

Escobar, A. (2003). Mundos y conocimientos de otro modo. El programa de investigación de modernidad/colonialidad latinoamericano. Tabula Rasa. 1: 51-86.

Fleck, L. (1986a). La génesis y el desarrollo de un hecho científico. Traducao de Luis Meana. Madrid: Alianza Editorial.

Fleck, L. (1986b). Some Specific features of the Medical Way of thinking 1927. In Cohen, R. S.; Sshanelle, T. (Eds.). Cognition and fact. Dodrecht. 39-46. 


\section{$5^{\circ}$ CONGRESO NACIONAL DE ENSEÑANZA DE LA FÍSICA. Universidad Pedagógica Nacional (U. P. N) \\ Universidad Distrital Francisco José de Caldas (U. D. F. J. C) Bogotá, Colombia. 16 al 20 de mayo 2011}

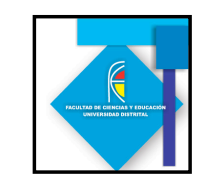

Giordan, A., \& De Vecchi, G. (1988). Los orígenes del saber. De la concepciones personales a los conceptos científicos. Sevilla: Diada Editores.

Grosfoguel, R. (2006). La descolonización de la economía política y los estudios postcoloniales: Transmodernidad, pensamiento fronterizo y colonialidad global. Tabula Rasa. 4:17-48.

Grosfoguel, R. (2005). The implications of subaltern epistemologies for global capitalism: Transmodernity, border thinking and global coloniaity- En William I. Robison y Rochard Applebaum (eds). Critical Globalization .

Grosfoguel, R. (2006b). World-System Analysis in the context of Transmodernity, Border Thimking and Glonal Coloniality. Review. 29.

Jhonson-Laird, P. (1996). Images, models, and propositional representational. In: De Vega et al. Models of visiospatial cognition. New York: University Press.

Johnson-Laird, P. (1983). Mental models. Cambridge: Harvard University.

Justi, R. (2006). La enseñanza de las ciencias basada en la elaboración de modelos.

Enseñanza de las ciencias. 24, 29: 173-184.

Justi, R., \& Gilbert, J. (2006). Models and Modelling in Chemical Education, en Gilbert, J., Jomg, O. D. Justi, R., Treagust, D. F. y V Driel, J. H. (Eds.). Chemical Education: Towards Research - based. Practice: Dordrecht: Kluwer. 47 -68.

McLaren, P. (1997). Pedagogía crítica y cultura depredadora. Políticas de oposición en la era posmoderna. Barcelona: Paidós.

Ministerio de Educación Nacional. Plan decenal de educación 2006-2010. (1994). Bogotá, Colombia.

Molina, A. (2002). Conglomerado de relevancias de niños y jóvenes. Científica. 4, 1: 187199.

Molina, A. (2005). El "otro" en la constitución de identidades culturalers. En Piedreita, C y Paredes, E (Editoras). Cultura política, identidades y nueva ciudadanía, Cúcuta, Sic Eitorial LTDA. 139-169.

Molina, A., López, D., \& Mójica, L. (2005). Ideas de los niños sobre la naturaleza: un estudio comparado. Revista Científica. 7(1): 41-62.

Moreira, M. A. (1994). Cambio conceptual: análisis crítico y propuestas a la luz de la teoría significativo. Greca, Ileana María. Cambio conceptual. Porto Alegre: Instituto de FísicaUFRGS Caixa. 
$5^{\circ}$ CONGRESO NACIONAL DE ENSEÑANZA DE LA FÍSICA.

Universidad Pedagógica Nacional (U. P. N)

Universidad Distrital Francisco José de Caldas (U. D. F. J. C)

Bogotá, Colombia. 16 al 20 de mayo 2011

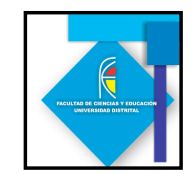

Moreira, M. A. (1999). Modelos mentales. Investigaciones en Enseñanza de las ciencias. 1, 3: 193-232.

Moreira, M. A. (2005). Representacoes mentais, modelos mentais e representacaos sociais. Porto Alegre: Universidad Federal Do Rio Grande Do Sul.

Moreira, M. A., \& Greca, I. (2003). Cambio Conceptual: Análisis crítico y propuestas a la luz de la teoría del aprendizaje significativo. Ciencia \& Educacao. 7-25.

Moreira, M. A., \& Greca, I. (2002). Modelos mentales y modelos conceptuales en la enseñanza y aprendizaje de las ciencias. Brasilera de Pesquisa em Educacao en Ciencias. 2, 3: 44-66.

Morrison, M., \& Morgan, M. (1999). Models as mediating instruments, en Morgan, M. S. y Morrison, M. (Eds.). Models as mediators, pp 10-37. Cambridge: University press.

Mortimer, E. (2000). Linguagem e Formacao de Conceitos no Ensino de Ciencias. Belo Horizonte: UFMG, MG.

Mortimer, E. (1998). Multivoicedness and univocality in classroom discourse: an example from theory of matter. International Journal for Science Education , 1: 67-82.

Mortimer, E. (2001). Perfil Conceptual: modos de penar y hablar en las aulas de ciencia. Infancia y Aprendizaje. 24, 4.

Norman, D. (1983). Some observations on mental modeles. En Gentner y Stevens, A.L. (Eds.). New York: Lawerence Erbaum Associates.

Nussbaum, J.-R. (1989). Classroom conceptual changa: philosophical perspectives. International Journal of Science Education. 11: 530-540.

Ortiz, H. (1995). Los conceptos de equilibrio y desequilibrio. Una alternativa a las formas explicativas en la Física. Tres casos concretos: Inercia, flotación y electromagnetismo. Bogotá, Colombia: Tesis de Maestría en Docencia de la Física. Universidad pedagógica Nacional.

Piaget, J. (1984). La representación del mundo en el niño. Madrid: Morata.

Piaget, J., \& Inheldert, B. (1972). De la lógica del niño a la lógica del adolescente. Buenos Aires: Paidós.

Pozo, J. I. (2003). ¿Puede la educación científica sustituir al saber cotidiano de los alumnos. II Congreso Iberoamericano de la Enseñanza de las Ciencias Experimentales. Madrid: Universidad de Alcalá. 
$5^{\circ}$ CONGRESO NACIONAL DE ENSEÑANZA DE LA FÍSICA.

Universidad Pedagógica Nacional (U. P. N)

Universidad Distrital Francisco José de Caldas (U. D. F. J. C)

Bogotá, Colombia. 16 al 20 de mayo 2011

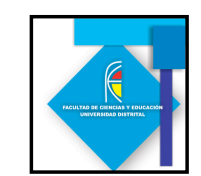

Pozo, J. I., \& Gómez, M. (1998). Aprender y enseñar ciencias. Del conocimiento cotidiano al conocimiento científico. Madrid: Morata.

Pozo, J. I., Rodríguez, A., \& Marrero, J. (1993). Las teorías implícitas. Una aproximación al pensamiento cotidiano. Madrid: Aprendizaje Visor.

Rodrigo, M. J. (1994). El hombre de las calles, el científico y el alumno: ¿Un solo constructivismo o tres? Investigación en la escuela. 23: 7-15.

Rodrigo, M. J. (1985). Las teorías implícitas en el conocimiento social. Infancia y aprendizaje. 31-34, 145-156.

Rodrigo, M., Rodríguez, A., \& Marrero, J. (1994). Las teorías implícitas. Una aproximación al conocimiento cotidiano. Madrid: Visor.

Utges, G., \& Pacca, J. (2003). Análisis factorial en la caracterización de representaciones implícitas. Reflexiones metodológicas a la luz de algunas investigaciones realizadas. Universidad del Rosario.

Utges, G., \& Pacca, J. (1998). Razonamiento analógico y aprendizaje significativo. Una discusión analizando analogías utilizadas en la enseñanza del concepto de onda. Atas do IV Encontro de Pesquisa em Ensino de Física. Florianópolis.

Utges, G., Jardón, A., Feráboli, L., \& Fernández, P. (2000). Teorías implícitas de los profesores sobre la tecnología y su enseñanza. Atas do VII Encontro de Pesquisa em Ensino de Física. Florionópolis.

Vergnaud, G. (2007). ¿En que sentido la teoría de los campos conceptuales puede ayudarnos para facilitar aprendizaje significativo? Investigacoes em Ensino de Ciencias. 12, 2: 285-302.

Vergnaud, G. (1996). A trama dos champs conceptuels na construcao dos connhecimientos. Revista do GEMPA. 4:9-19.

Vergnaud, G. (1990). La theorie des champs conceptuels. Resecherches. Didactique des Mathematiques. 10, 23: 133-170. 